$\underline{\text { Review Article }}$

\title{
A PRESPECTIVE STUDY ON APOBEC PROTEIN FAMILY
}

\author{
S. GEJALAKSHMI ${ }^{*}$, N. HARIKRISHNAN ${ }^{1}$, S. KOMAL
}

Faculty of Pharmacy, Department of Pharmaceutical Chemistry Dr. M. G. R. Educational and Research Institute, Velappanchavadi, Chennai-77

Email: gejampharm@gmail.com

Received: 12 Aug 2019, Revised and Accepted: 10 Oct 2019

\begin{abstract}
Apobec is an apolipoprotein B mRNA editing enzyme, catalytic polypeptide-like" is a family of deaminases. It has two types of APOBEC enzymes n-terminal of apobec enzyme and C-terminal of APOBEC enzyme. N-terminal domain is catalytic domain and C-terminal domain is a pseudo catalytic domain. Pathogen and cellular genome undergo mutation by human DNA cytosine to uracil deaminases. Three subtypes of APOBEC3D. APOBEC3F, APOBEC3G and APOBEC3H restrict human deficiency virus-1. Two APOBEC enzymes are the sources of somatic mutagenesis in cancer cells that drive tumor evolution and manifest clinically as theraphy resistance. This review of the APOBEC family will focus on an open question in regulation, namely what role the interactions of these proteins with RNA have in editing substrate recognition or allosteric regulation of DNA mutagenic and host-defense activities.
\end{abstract}

Keywords: APOBEC, DNA mutagen, m-RNA

(C) 2019 The Authors. Published by Innovare Academic Sciences Pvt Ltd. This is an open access article under the CC BY license (http://creativecommons.org/licenses/by/4.0/] DOI: http://dx.doi.org/10.22159/ijcpr.2019v11i6.36345

\section{INTRODUCTION}

Mutation results in genomic variation causes beneficial, neutral or harmful sequences for an organism. Geneomic mutation results in DNA replication errors such as mis incorporation of a nucleotide by a polymerase and environmental mutagens, such as certain chemicals and ionizing radiation. Certain innate enzymatic sources of mutation in humans with significant roles in diseases. Human DNA has 11 polypeptide $C$ to $U$ editing enzymes including activation induced deaminase. This review focus on the role of apobec enzyme to target viral or cancer cells.

Structure mechanism and enzymatic activity of human A3 Enzymes: The majority of human cancers display one or more patterns of genomic instability, ranging from large-scale changes in chromosome architecture and tumor karyotype through to multitudes of single nucleotide mutations. Diversity created by these seemingly chaotic processes provides the substrate for selection within tumors. The relative importance of selection and elevated mutation rates (when used in its broadest sense covers both large scale chromosomal and single nucleotide changes) has been a subject of much debate. APOBEC enzymes-major contributors to cancer mutagenesis the largest newly defined source of mutation in many different tumor types is enzymatic DNA cytosine deamination by several members of the APOBEC family (reviewed recently in [15]. These enzymes were identified independently in 2002 as DNA mutators and as anti-viral factors [6-8]. Apart from activationinduced deaminase (AID), which deaminates expressed antibody genes, all of the family members are named after the founder, APOBEC1, which edits both single-stranded DNA cytosines and cellular mRNA cytosines (as implied by its rarely used full name: apolipoprotein B mRNA editing enzyme catalytic polypeptide 1). Importantly, the sheer existence of multiple DNA mutating enzymes in human cells strongly suggested that at least one could become deregulated and promote mutagenesis in cancer [9]. However, dominant roles in antiviral innate immunity and particularly in HIV1 restriction took center stage for most of the following decade as technologies and experimental systems have had to be developed to test the cancer hypothesis [10].

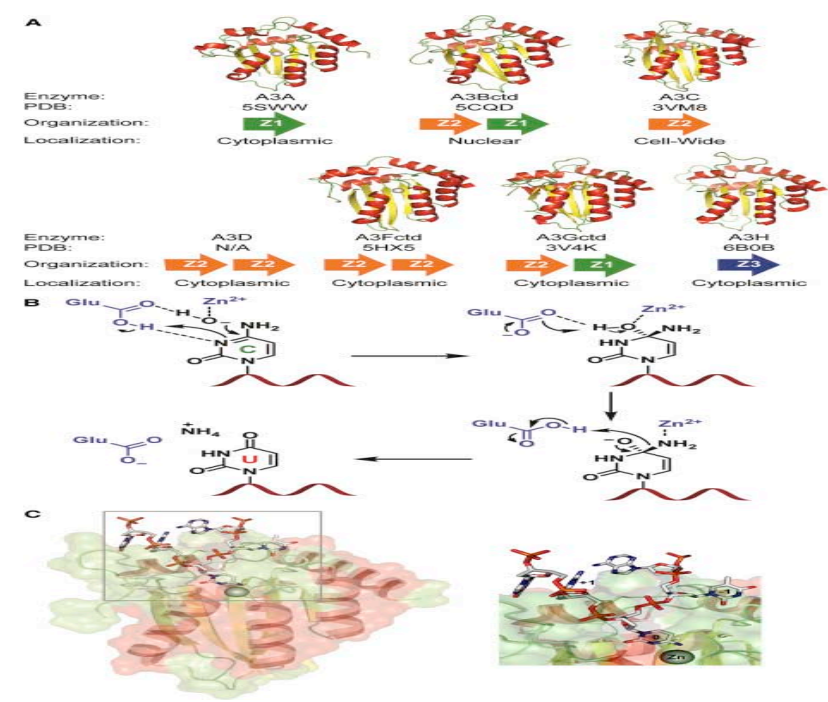

Fig. 1: However, dominant roles in antiviral innate immunity and particularly in HIV-1 restriction took center stage for most of the following decade as technologies and experimental systems have had to be developed to test the cancer hypothesis 
Madison B. Adolph, Robin P. Love, Linda Chelico [9]. The Apolipoprotein B mRNA editing complex (APOBEC) family of enzymes contains single-stranded polynucleotide cytidine deaminases. These enzymes catalyze the deamination of cytidine in RNA or single-stranded DNA, which forms uracil. From this 11 member enzyme family in humans, the deamination of singlestranded DNA by the seven APOBEC3 family members is considered here. The APOBEC3 family has many roles, such as restricting endogenous and exogenous retrovirus replication and retrotransposon insertion events and reducing DNA-induced inflammation. Similar to other APOBEC family members, the APOBEC3 enzymes are a double-edged sword that can catalyze deamination of cytosine in genomic DNA, which results in potential genomic instability due to the many mutagenic fates of uracil in DNA. Here, we discuss how these enzymes find their single-stranded DNA substrate in different biological contexts such as during human immunodeficiency virus (HIV) proviral DNA synthesis, retrotransposition of the LINE-1 element, and the "off-target" genomic DNA substrate. The enzymes must be able to efficiently deaminate transiently available single-stranded DNA during reverse transcription, replication, or transcription. Specific biochemical characteristics promote deamination in each situation to increase enzyme efficiency through processivity, rapid enzyme cycling between substrates, or oligomerization state. The use of biochemical data to clarify biological functions and alignment with cellular data is discussed. Models to bridge knowledge from biochemical, structural, and single molecule experiments are presented.

Jeffrey Chen, Thomas MacCarthy [10] designed the study of the AID/APOBEC genes are a family of cytidine deaminases that have evolved in vertebrates, and particularly mammals, to mutate RNA and DNA at distinct preferred nucleotide contexts (or ${ }^{a}$ hotspots ${ }^{\circ}$ ) on foreign genomes such as viruses and retrotransposons. These enzymes play a pivotal role in intrinsic immunity defense mechanisms, often deleteriously mutating invading retroviruses or retrotransposons and, in the case of AID, changing antibody sequences to drive affinity maturation. We investigate the strength of various hotspots on their known biological targets by evaluating the potential impact of mutations on the DNA coding sequences of these targets, and compare these results to hypothetical hotspots that did not evolve. We find that the existing AID/APOBEC hotspots have a large impact on \retrotransposons and non-mammalian viruses while having a much smaller effect on vital mammalian genes, suggesting co-evolution with AID/APOBECs may have had an impact on the genomes of the viruses we analyzed. We determine that GC content appears to be a significant, but not sole, factor in resistance to deaminase activity. We discuss possible mechanisms AID and APOBEC viral targets have adopted to escape the impacts of deamination activity, including changing the GC content of the genome.

Shraddha Sharma [11] APOBEC3G is a cytidine deaminase with two homologous domains and restricts retroelements and HIV-1. APOBEC3G deaminates single-stranded DNAs via its C-terminal domain, whereas the $\mathrm{N}$-terminal domain is considered non-catalytic. Although APOBEC3G is known to bind RNAs, APOBEC3G-mediated RNA editing has not been observed. We recently discovered RNA editing by the single-domain enzyme APOBEC3A in innate immune cells. To determine if APOBEC3G is capable of RNA editing, we transiently expressed APOBEC3G in the HEK293T cell line and performed transcriptome-wide RNA sequencing. We show that APOBEC3G causes site-specific C-to-U editing of mRNAs from over 600 genes. The edited cytidines are often flanked by inverted repeats, but are largely distinct from those deaminated by APOBEC3A. We verified protein-recoding RNA editing of selected genes including several that are known to be involved in HIV-1 infectivity. APOBEC3G co-purifies with highly edited mRNA substrates. We find that conserved catalytic residues in both cytidine deaminase domains are required for RNA editing. Our findings demonstrate the novel RNA editing function of APOBEC3G and suggest a role for the $\mathrm{N}$-terminal domain in RNA editing.

Spyridon Stavrou [12] has decribed Apolipoprotein B editing complex 3 family members are cytidine deaminases that play important roles inintrinsic responses to infection by retroviruses and have been implicated in the control of other viruses, such as parvoviruses, herpesviruses, papillomaviruses, hepatitis B virus, and retrotransposons.

Although their direct effect on modification of viral DNA has been clearly demonstrated, whether they play additional roles in innate and adaptive immunity to viruses is less clear. We review the data regarding the various steps in the innate and adaptive immune response to virus infection in which apolipoprotein B editing complex 3 proteins have been implicated.

Kimberly M. Prohaska [13] has proven the Cytidine deaminases have important roles in the regulation of nucleoside/deoxynucleoside pools for DNA and RNA synthesis. The APOBEC family of cytidine deaminases (named after the first member of the family that was described, Apolipoprotein B mRNA Editing Catalytic Subunit 1, also known as APOBEC1 or A1) is a fascinating group of mutagenic proteins that use RNA and single-stranded DNA (ssDNA) as substrates for their cytidine or deoxycytidine deaminase activities. APOBEC proteins and base-modification nucleic acid editing have been the subject of numerous publications, reviews, and speculation. These proteins play diverse roles in host cell defense, protecting cells from invading genetic material, enabling the acquired immune response to antigens and changing protein expression at the level of the genetic code in mRNA or DNA.|The amazing power these proteins have for interphase cell functions relies onstructural and biochemical properties that are beginning to be understood. Atthe same time, the substrate selectivity of each member in the family and their regulation remains to be elucidated. This review of the APOBEC family will focus on an open question in regulation, namely what role the interactions of these proteins with RNA have in editing substrate recognition or allosteric regulation of DNA mutagenic and host-defense activities.

Naveenan Navaratnam [14] determined Enzymes that deaminate cytidine to uridine play an important role in a variety of pathways from bacteria to man. Ancestral members of this family were able to deaminate cytidine only in a mononucleotide or nucleoside context. Recently, a family of enzymes has been discovered with the ability to deaminate cytidines on RNA or DNA. The first member of this new family is APOBEC1, which deaminates apolipoprotein B messenger RNA to generate a premature stop codon. APOBEC1 has the conserved active site motif found in Escherichia coli cytidine deaminase. In addition, APOBEC1 has a unique motif containing 2 phenylalanine residues and an insert of 4 amino acid residues across the active site motif. This motif is present in APOBEC family members including activation-induced cytidine deaminase (AID), APOBEC2, and APOBEC3A through APOBEC3G. AID is essential for initiating class-switch recombination, somatic hypermutation, and gene conversion. The APOBEC3 family is unique to primates. APOBEC3G is able to protect cells from human immunodeficiency virus and other viral infections. This function is not unique to APOBEC3G; other APOBEC3 family members also have this ability. Overexpression of enzymes in this family can cause cancer, suggesting that the genes for the APOBEC family of proteins are proto-oncogenes.

Jianlong Gao [15] has discovered that Cancer is currently viewed as a disease of evolving genomic instability and abnormal epigenomic modifications. Most solid cancers harbor oncogenic gene mutations driven by both extrinsic and intrinsic factors. Apolipoprotein B mRNA editing catalytic polypeptide-like family (APOBEC) enzymes have an intrinsic deamination activity to convert cytosine to uracil during RNA editing and retrovirus or retrotransposon restriction. Beyond their natural defense in innate immunity, compelling evidence showed that a subclass of APOBEC 3 can cause high mutation burden in various types of cancer genomes, and high expression subtypes of APOBEC3 may contribute to drug resistance and associate with clinical outcomes. The underlying molecular mechanisms of APOBECmediated hypermutation phenotype are poorly understood. In this review, we discuss the linkage of activation-induced deaminase (AID)/APOBEC3 enzymes to tumorigenesis, highlight the dysregulatory mechanisms of APOBEC3 activities during cancer development, and propose potential approaches to targeting APOBEC3-mediated mutagenesis for cancer interventions. 
Benjamin JM [16] has underwent a study on breast cancer genomes have revealed a novel form of mutation showers (kataegis) in which multiple same-strand substitutions at C: G pairs spaced one to several hundred nucleotides apart are clustered over kilobase-sized regions, often associated with sites of DNA rearrangement. We show kataegis can result from AID/APOBEC-catalysed cytidine deamination in the vicinity of DNA breaks, likely through action on single-stranded DNA exposed during resection. Cancer-like kataegis can be recapitulated by expression of AID/APOBEC family deaminases in yeast where it largely depends on uracil excision, which generates an abasic site for strand breakage. Localized kataegis can also be nucleated by an I-SceI-induced break. Genomewide patterns of APOBEC3-catalyzed deamination in yeast reveal $\mathrm{APOBEC} 3 \mathrm{~B}$ and $3 \mathrm{~A}$ as the deaminases whose mutational signatures are most similar to those of breast cancer kataegic mutations. Together with expression and functional assays, the results implicate APOBEC3B/A in breast cancer hypermutation and give insight into the mechanism of kataegis.

Sara L. Sawyer [17] Host genomes have adopted several strategies to curb the proliferation of transposable elements and viruses. Arecently discovered novel primate defense against retroviral infection involves a single-stranded DNA-editing enzyme, APOBEC3G that causes hypermutation of HIV. The HIV-encoded virion infectivity factor (Vif) protein targets APOBEC3G for destruction, setting up a genetic conflict between the APOBEC3G and Vif genes. This kind of conflict leads to rapid fixation of mutations that alter amino acids at the protein-protein interface, referred to as positive selection. We show that the APOBEC3G gene has been subject to strong positive selection throughout the history of primate evolution. Unexpectedly, this selection appears more ancient than, and is likely only partially caused by, modern lentiviruses. Furthermore, five additional APOBEC genes in the human genome appear to be engaged in similar genetic conflicts, displaying some of the highest signals for positive selection in the human genome. Despite being only recently discovered, editing of RNA and DNA may thus represent an ancient form of host defense in primate genomes.

Stephen J. Hollanda [18] Cytidine deaminases of the AID/APOBEC family catalyze C-to-U nucleotide transitions in mRNA or DNA. Members of the APOBEC3 branch are involved in antiviral defense, whereas AID contributes to diversification of antibody repertoires in jawed vertebrates via somatic hypermutation, gene conversion, and class switch recombination.

In the extant jawless vertebrate, the lamprey, two members of the AID/APOBEC family are implicated in the generation of somatic diversity of the variable lymphocyte receptors (VLRs). Expression studies linked CDA1 and CDA2 genes to the assembly of VLRA/C genes in T-like cells and the VLRB genes in B-like cells, respectively. Here, we identify and characterize several CDA1-like genes in the larvae of different lamprey species and demonstrate that these encode active cytidine deaminases. Structural comparisons of theCDA1 variants highlighted substantial differences in surface charge; this observation is supported by our finding that the enzymes require different conditions and substrates for optimal activity in vitro. Strikingly,we also found that the number of CDA-like genes present in individuals of the same species is variable. Nevertheless, irrespective of the number of different CDA1-like genes present, all lamprey larvae have at least one functional CDA1related gene encoding an enzyme with predicted structural and chemical features generally comparable to jawed vertebrate AID. Our findings suggest that, similar to APOBEC3 branch expansion in jawed vertebrates, the AID/APOBEC family has undergone substantial diversification in lamprey, possibly indicative of multiple distinct biological roles.

Shraddha Sharma [19], Protein recoding by RNA editing is required for normal health and evolutionary adaptation. However, de novo induction of RNA editing in response to environmental factors is an uncommon phenomenon. While APOBEC3A edits many mRNAs in monocytes/macrophages in response to hypoxia and interferons, the physiological significance of such editing is unclear. Here we show that the related APOBEC3G cytidine deaminase induces site-specific C-to-
URNA editing in natural killer (NK), CD8+T cells and lymphoma cell lines upon cellular crowding and hypoxia. RNASeq analysis of hypoxic $\mathrm{NK}$ cells reveals widespread $\mathrm{C}$-to-U recoding mRNAediting that is enriched for genes involved in mRNA translation. APOBEC3G promotes Warburg like metabolic remodeling and reduces proliferation of HuT78 T cells under similar conditions.

\section{Summary and future}

\section{Perspectives}

Cancer is currently viewed as a disease of evolving genomic instability and abnormal epigenomic modifications. endogenous mutagens also cause cancer, such as $A P O B E C$ family genes, are found to associate with cancer development,and their high expressions correlate to mutational burden in certain types of cancer and leads to death. Several studies indicate that NF-jB pathway activation, p53 inactivationby HPV oncoprotein E6/E7 activation or loss-of-function mutations in the TP53 gene and replication stress activation are directors for transcriptional activation of APOBEC, in particular, APOBEC3B. We reviewed different strategies to directly or indirectly target $\mathrm{APOBEC} 3 \mathrm{~B}$ as a prototype regimen for inactivation of Summary of apolipoprotein B mRNA editing enzyme catalytic polypeptide-like (APOBEC) 3B transcriptional regulations in cancer cells. Among all APOBEC3B is the main source of mutagenesis in multiple types of cancer. High expression of APOBEC3B and increased activity have been reported in HER2+breast cancer, human papillomavirus (HPV)+cervical cancer, and head and neck squamous carcinoma. Nuclear factor kappa B (NF-jB) pathway activation, 53 DNA replication stress54 and HPV E6/7 oncoproteins and mutant p5352 are able to turn on APOBEC3B expression. In contrast, WT p53 activation suppresses APOBEC3B expression through p21-mediated transcriptional suppressive complexes occupancy of promoter region of APOBEC3B.51, 52 AID, activationinduced deaminase; IFN, interferon; TNF, tumor necrosis factor aberrant expression of APOBEC for potential interventions. We are only beginning to appreciate the effects of APOBEC-mediated mutagenesis in cancer; many questions remain to be answered. For example, whether elevated expressions of APOBEC3 are causative factors for cancer initiation or just a bystander consequence during cancer development; how can we effectively prevent the off-target harmful mutations by APOBEC at the precancerous stage; why elevated expression of APOBEC has a paradoxically clinical benefit on cancers; and how can we quickly apply hypothetical approaches to manage subtypes of cancer patients with aberrant APOBEC expression in the clinical setting. With more research focused on APOBEC biology. We conclude that current review will be addresses in the near future for the development of new drug for cancer treatment.

\section{AUTHORS CONTRIBUTIONS}

All the author have contributed equally.

\section{CONFLICT OF INTERESTS}

\section{Declared none}

\section{REFERENCES}

1. Gerlinger M, McGranahan N, Dewhurst SM, Burrell RA, Tomlinson I, Swanton C. Cancer: evolution within a lifetime. Ann Rev Gen 2014;48:215-36.

2. Roberts SA, Gordenin DA. Hypermutation in human cancer genomes: footprints and mechanisms. Nat Rev Cancer 2014;14:786-800.

3. Burns MB, Leonard B, Harris RS. APOBEC3B: pathological consequences of an innate immune DNA mutator. Biomed J 2015;38:102-10.

4. Henderson S, Fenton T. APOBEC3 genes: retroviral restriction factors to cancer drivers. Trends Mol Med 2015;21:274-84.

5. Harris RS. Molecular mechanism and clinical impact of APOBEC3B-catalyzed mutagenesis in breast cancer. Breast Cancer Res 2015;17:8.

6. Harris RS, Petersen Mahrt SK, Neuberger MS. RNA editing enzyme APOBEC1 and some of its homologs can act as DNA mutators. Mol Cell 2002;10:1247-53. 
7. Petersen Mahrt SK, Harris RS, Neuberger MS. AID mutates E. coli suggesting a DNA deamination mechanism for antibody diversification. Nature 2002;418:99-103.

8. Sheehy AM, Gaddis NC, Choi JD, Malim MH. Isolation of a human gene that inhibits HIV-1 infection and is suppressed by the viral Vif protein. Nature 2002;418:646-50.

9. Madison B Adolph, Robin P Love, Linda Chelico. Apobec enzymes as targets for virus and cancer therapy. Cell Chem Biol 2018;25:36-49.

10. Jeffrey Chen, Thomas MacCarthy. The preferred nucleotide contexts of the AID/APOBEC cytidine deaminases have differential effects when mutating retrotransposon and virus sequences compared to host genes. PLOS Comput Biol 2017;13:e1005471.

11. Shraddha Sharma, Santosh K Patnaik, Robert T Taggart, Bora E Baysal. The double-domain cytidine deaminase APOBEC3G is a cellular site-specific RNA editing enzyme. Sci Rep 2016;6:39100.

12. Uqing Feng, Tayyba T Baig, Robin P Love, Linda Chelico, Yuqing Feng, Tayyba T Baig, et al. Suppression of APOBEC3-mediated restriction of HIV. Front Microbiol 2014;5:450.

13. Spyridon Stavrou, Susan R. Ross APOBEC3 proteins in viral immunity. J Immunol 2015;195:4565-70.

14. Kimberly M Prohaska, Ryan P Bennett, Jason D Salter, Harold C Smith. The multifaceted roles of RNAbinding in APOBEC cytidinedeaminase functions. Wiley Interdiscip Rev RNA 2014;5:493-508.
15. Naveenan Navaratnam. Rizwan sarwar an overview of cytidine deaminases. Int J Hematol 2006;83:195-200.

16. Jianlong Gao, Hani Choudhry, Wei Cao. Apolipoprotein B mRNA editing enzyme catalytic polypeptide-like family genes activation and regulation during tumorigenesis. Cancer Sci 2018;109:2375-82

17. Benjamin JM Taylor, Serena Nik-Zainal, Yee Ling Wu, Lucy A Stebbings, Keiran Raine, Peter I Campbell, Cristina Rada, et al. DNA deaminases induce break-associated mutation showers with implication of $\mathrm{APOBEC} 3 \mathrm{~B}$ and $3 \mathrm{~A}$ in breast cancer kataegis. Elife 2013;2:e00534.

18. Sara L Sawyer, Michael Emerman, Harmit S Malik. Ancient adaptive evolution of the primate antiviral DNA-editing enzyme APOBEC3G. PLoS Biol 2004;2:e275.

19. Stephen J Hollanda, Lesley M Berghuisb, Justin J Kingb, Lakshminarayan M Iyerc, Katarzyna Sikoraa. Expansions, diversification, and interindividual copy number variations of AID/APOBEC family cytidine deaminase genes in lampreys. PLoS Biol 2004;115:E3211-E3220.

20. Stephen J Hollanda, Lesley M Berghuisb, Justin J Kingb, Lakshminarayan M Iyerc, Katarzyna Sikoraa, Heather Fifieldb, et al. Expansions, diversification, and interindividual copy number variations of AID/APOBEC family cytidine deaminase genes in lampreys. PNAS 2018;115:E3211-E3220.

21. Shraddha Sharma, Jianmin Wang, Scott Portwood, Eduardo Cortes-Gomez, Orla Maguire. Mitochondrial hypoxic stress induces widespread RNA editing by 1 APOBEC3G in lymphocytes Genome Biol 2019;20:37. 